\title{
Assessing the association between bronchiolitis in infancy and recurrent wheeze: a whole English birth cohort case-control study
}

\author{
Robin Marlow, Adam Finn, John Henderson
}

Population Health Sciences, University of Bristol, Bristol, UK

\section{Correspondence to} Dr Robin Marlow, Population Health Sciences, University of Bristol, Bristol BS2 8AE, UK; robin.marlow@bristol.ac.uk

Received 14 June 2018 Revised 8 November 2018 Accepted 26 November 2018 Published Online First 4 April 2019

\section{Linked}

http://dx.doi.org/10.1136/ thoraxjnl-2018-212814

\section{Check for updates}

(C) Author(s) (or their employer(s)) 2019. No commercial re-use. See rights and permissions. Published by BMJ.

To cite: Marlow R, Finn A, Henderson J. Thorax

2019:74:503-505.

\section{ABSTRACT}

The precise association between bronchiolitis and predisposition to childhood wheeze is unclear. We assessed bronchiolitis aetiology and later wheeze phenotypes in the entire 2007 English birth cohort. All infants admitted to hospital in England during their first year of life with bronchiolitis or urinary tract infection (UTI) were followed using Hospital Episode Statistics to determine risk and characteristics of wheeze admission over the subsequent 8 years. In our cohort of 21272 children compared with UTI, the risk of wheeze admission was higher with previous bronchiolitis (risk ratio (RR) 2.4), even higher in those with non-respiratory syncytial virus bronchiolitis (RR 3.1) and persisted into late-onset wheeze (RR 1.7).

\section{INTRODUCTION}

Bronchiolitis is the most common reason for hospital admission in infancy. In the UK, currently $4 \%$ of the children born per year are admitted to hospital with bronchiolitis $(40.4 / 1000) .{ }^{1}$ Wheeze is one of the the most common causes for admission in later childhood-3.1 admissions/1000 children in 2016. ${ }^{2}$ These two respiratory diagnoses combined represent a significant burden for both children, their families and the health service. ${ }^{3}$ The link between respiratory syncytial virus (RSV) bronchiolitis and early (post bronchiolitis) wheeze is recognised; however, the association with late phenotypes of wheeze and other viruses is less clear. ${ }^{4}$ With potential vaccines and therapeutic agents in development against RSV, accurately quantifying any potentially causal link could be of use for their health economic assessment. In this study, we sought to characterise the association between different viral causes of bronchiolitis and phenotypes of childhood wheeze.

\section{METHOD}

Hospital Episode Statistics discharge codes were used to create two cohorts; all infants $(<12$ months of age) admitted to hospital in England during 2007 diagnosed with bronchiolitis or as a comparator with a urinary tract infection (UTI-chosen as the the most common non-respiratory cause for infant admission to hospital). ${ }^{2}$ Our case definition of bronchiolitis was children $<12$ months of age admitted to hospital with a clinician diagnosis of bronchiolitis (table 1). The all-cause bronchiolitis cohort was subdivided by coded causative organism (RSV, non-RSV (nRSV) or unspecified bronchiolitis (usBr)). Patients with both diagnoses during the first year of life were excluded as were all those who had been admitted to neonatal intensive care at birth (due to an increased risk of chronic lung disease). From their first birthday, we compared the number and pattern of admissions with wheeze between the two cohorts over the subsequent 8 years. Timings of recurrent wheeze episodes were used to categorise patients into previously described wheeze phenotypes. ${ }^{5}$ To test for confounding between our cohorts, we compared the rates of head injury (a common non-infective reason for paediatric admission) as a negative control, with the hypothesis that if unbiased that this should not differ between cohorts. ${ }^{6}$ R V.3.4.1 was used for data cleaning and to perform log-binomial regression adjusted for gender to calculate relative risks.

\section{RESULTS}

From the 2007 birth cohort of 642000 children, we identified 17946 cases of bronchiolitis and 3326 of UTI (table 1) with risks of later admission with any wheeze of 19\%-7\%, respectively; risk ratio (RR) of admission 2.4 (95\% CI 2.2 to 2.8). As there was a significant difference in gender between the cohorts, $60 \%$ male in bronchiolitis versus $48 \%$ male in UTI, we adjusted for gender in our models. Our negative control suggested no bias with no difference found in the risk of head injury admission (RR $0.96,95 \% \mathrm{CI} 0.7$ to 1.3 ) between the cohorts.

Against all-cause wheeze admissions, nRSV bronchiolitis had a significantly stronger association, RR 3.1 (2.4-3.9), than for RSV, RR 2.3 (2.0-2.6) $\mathrm{p}=0.01$, but not the undifferentiated usBr, RR 2.5 (2.2-2.8) $\mathrm{p}=0.06$. Using length of bronchiolitis

\begin{tabular}{|c|c|c|c|}
\hline Groups & & $\mathrm{N}$ & ICD-10 codes \\
\hline \multirow{3}{*}{$\begin{array}{l}\text { Bronchiolitis } \\
\text { (60\% male) }\end{array}$} & RSV & 4399 & $\mathrm{~J} 12.1, \mathrm{~J} 21.0$ \\
\hline & Non-RSV & 243 & $\begin{array}{l}\mathrm{J} 12.0, \mathrm{~J} 12.2 \\
\mathrm{~J} 12.3, \mathrm{~J} 12.8 \\
\mathrm{~J} 21.1, \mathrm{~J} 21.8\end{array}$ \\
\hline & Unspecified & 13304 & $J 12.9, J 21.9$ \\
\hline \multicolumn{2}{|c|}{ UTI (control) ( $52 \%$ male) } & 3326 & N39.0 \\
\hline \multirow[t]{2}{*}{ Outcomes } & Wheezing & 4472 & $\begin{array}{l}\text { J45. }{ }^{*}, J 46^{*}, \\
\text { R06.2 }\end{array}$ \\
\hline & Head injury & 325 & S09* \\
\hline \multirow[t]{2}{*}{ Excluded } & $\begin{array}{l}\text { Neonatal } \\
\text { admissions }\end{array}$ & 4654 & NA \\
\hline & Both diagnoses & 191 & NA \\
\hline
\end{tabular}

ICD-10, International Classification of Diseases, 10th revision; RS respiratory syncytial virus; NA, not applicable; UTI, urinary tract infection. 
Table 2 Risk ratio (RR) of later admission with wheeze by aetiology and length of bronchiolitis admission

\begin{tabular}{lllllll}
\hline Length of admission (days) & 0 & $1->3$ & $4->6$ & 7 to 13 & $>14$ & Combined \\
\hline All bronchiolitis RR $(95 \% \mathrm{Cl})$ & $2.16(1.9$ to 2.5$)$ & $2.37(2.1$ to 2.7$)$ & $2.57(2.3$ to 2.9$)$ & $3.37(2.9$ to 3.9$)$ & $3.24(2.4$ to 4.3$)$ & $2.4(2.2$ to 2.8$)$ \\
RSV RR $(95 \% \mathrm{Cl})$ & $1.6(1.2$ to 2.2$)$ & $2.0(1.7$ to 2.4$)$ & $2.3(1.7$ to 2.4$)$ & $3.1(2.6$ to 3.7$)$ & $2.9(1.8$ to 4.2$)$ & $2.3(2.0$ to 2.6$)$ \\
nRSV RR $(95 \% \mathrm{Cl})$ & $2.5(0.8$ to 5.2$)$ & $1.5(0.7$ to 2.6$)$ & 3.52 .3 to 4.9$)$ & $5(3.3$ to 7.0$)$ & $3.6(1.4$ to 6.6$)$ & $3.1(2.4$ to 4.0$)$ \\
usBr RR $(95 \% \mathrm{Cl})$ & $2.2(1.9$ to 2.5$)$ & $2.52(2.2$ to 2.8$)$ & $2.7(2.4$ to 3.1$)$ & $3.5(2.9$ to 4.3$)$ & $3.7(2.3$ to 5.5$)$ & $2.5(2.2$ to 2.8$)$ \\
\hline
\end{tabular}

nRSV, non-respiratory syncytial virus; us $B r$, unspecified bronchiolitis.

admission as a proxy for disease severity there was an apparent dose effect, with risk of later wheeze increased with admissions $<24$ hours and steadily increasing for longer admissions (table 2). All-cause bronchiolitis increased the risk of all phenotypes of wheeze, particularly transient, persistent early and persistent (table 3).

\section{DISCUSSION}

We have shown that significantly more cases of all phenotypes of wheeze occur in children that have had bronchiolitis in infancy compared with those who had a UTI, with the largest effects seen in long admissions with non-RSV bronchiolitis. This effect is most marked for the early wheeze phenotypes but persists for even late-onset wheeze.

While the link between RSV bronchiolitis and later respiratory pathology has been consistently demonstrated, the strength and details of the association vary between populations and has not been previously described in a UK cohort. ${ }^{7}$ Studies have also linked rhinovirus, human meta-pneumovirus and parainfluenzae as associated with later wheeze, with some suggesting a stronger effect for non-RSV bronchiolitis. ${ }^{8}$ The strongest form of evidence for assessing the association between bronchiolitis and wheeze is from randomised controlled trials (RCTs) of monoclonal antibodies against RSV. The Dutch MAKI RCT of paluvizumab in premature infants found that at 6 years, there was a significant reduction in parentally reported wheeze $(11.6 \%$ vs $19.9 \%)$ but not in physician diagnosed $(10.3 \%$ vs $9.9 \%)$, concluding that there was no clinically significant effect. ${ }^{9}$ The phase III RCT of motavizumab in 2170 term Navajo infants found an $87 \%$ relative reduction in RSV-related admissions but no effect on rates of medically attended wheezing (14.9\% vs $14.0 \%)$ during $1-3$ years of follow-up. ${ }^{10}$ In our cohorts, rates of bronchiolitis-associated late-onset wheeze were low (5\% vs $3 \%)$, raising the possibility that with $<1000$ patients per arm and only a subset of these developing bronchiolitis, these trials were not adequately powered to detect this difference. Although a small percentage change, we would argue that on a population scale, a vaccine that prevented any cases of a chronic health condition such as asthma as a secondary gain would be significant.

The strengths of this paper are that it follows a whole English population birth cohort of 642000 children over an 8 -year period. Using a hard outcome of admission rather than parentally reported or clinician diagnosed wheeze, it characterises the burden of admissions due to different phenotypes of wheeze. Our primary analysis using all diagnostic fields found similar frequencies of wheezing during admission as in parentally reported community surveys, ${ }^{11}$ restricting analysis to the primary diagnostic code found reduced incidence of wheeze $(7.8 \%$ post bronchiolitis vs $3.1 \%$ post UTI) but with a similar RR $2.4(2.0-3.0)$ between the groups. Its limitations are that it is reliant on quality of diagnostic coding, rates of routine virological testing and could not assess the effects on mild non-admitted wheeze presentations. As a retrospective analysis of routine health data, we were not able to distinguish between different syndromic phenotypes of bronchiolitis as our dataset limited us to identifying patients by diagnostic clinical coding. Rates of routine virological testing carried out in the UK in 2007 meant we could only meaningfully compare RSV with non-RSV subgroups. Our cohort of hospitalised children represent a severe subgroup and thus our findings are likely to be an overestimate of true population effect. Our analysis was controlled for gender; in a sensitivity analysis, we also included the index of multiple deprivations, but this did not alter the RR estimates. There may be other biases that we were not able to identify. Fundamentally, as an observational study design, we cannot hope to prove causality and must be cautious that our findings are not a product of the phenotype categorisation. ${ }^{12}$

While the narrative that bronchiolitis leads to an inflammatory state, the start of the allergic march and predisposition to later wheeze is appealing; that all causes of bronchiolitis are associated with all phenotypes of wheeze suggests this interpretation is actually the cart leading the horse. Perhaps bronchiolitis is simply a marker of children who are likely to go on to wheeze in later life and represents the sentinel manifestation of those genetically or

Table 3 Distribution of wheeze phenotypes for different cohorts and resulting risk ratios (RRs)

\begin{tabular}{|c|c|c|c|c|c|c|c|}
\hline \multirow[b]{2}{*}{ Wheeze } & \multirow[b]{2}{*}{ All wheeze } & \multicolumn{6}{|c|}{ Phenotype } \\
\hline & & NEVER & TREY & PEEY & INTER & LATE & PRST \\
\hline Bronchiolitis & $18.7 \%$ & $80.1 \%$ & $4.4 \%$ & $0.8 \%$ & $7.6 \%$ & $5.0 \%$ & $1.0 \%$ \\
\hline $\mathrm{RR}$ & 2.43 & NA & 5.4 & 4.8 & 2.6 & 1.7 & 5.2 \\
\hline $95 \% \mathrm{Cl}$ & 2.2 to 2.8 & NA & 3.8 to 8.0 & 2.3 to 12.1 & 2.1 to 3.1 & 1.4 to 2.1 & 2.6 to 12.2 \\
\hline
\end{tabular}

Phenotypes: NEVER — no wheeze; PRST—persistent, wheeze before 18 months until > 69 months; TREY — transient early, wheeze before 18 months until 42 months; PEEYprolonged early, wheeze before 18 months until 69 months; INTER -intermediate, no wheeze until after 42 months; LATE-late onset, no wheeze until after 69 months. NA, not applicable; UTI, urinary tract infection. 
environmentally predisposed to wheeze. That the association for early wheeze is stronger implies that this is more complicated than the revealing of an innate state. It may be that different clinical phenotypes of bronchiolitis ${ }^{13}$ would be helpful in this prognostication, but we were not able to assess this using this dataset.

Fundamentally, these data highlight our inability to prospectively clinically distinguish between wheeze phenotypes. It is reassuring that $80 \%$ of children with even severe bronchiolitis do not go on to wheeze. Academic interest aside, the reason for this study is to be able to answer parents' questions of 'will this happen again?' Although these data cannot elucidate the cause, they are helpful to describe likely respiratory trajectories for children. We now plan to expand this research by also examining wheeze attendances in the emergency department and primary care to see if the association still holds for the less severe cases of bronchiolitis.

\section{Twitter @robindmarlow}

Contributors RM contributed to the study design, analysis and authorship of the first draft. AF and JH critically appraised and developed the manuscript.

Funding RM is funded by the NIHR as an Academic Clinical Lecturer and is a member of the NIHR Health Protection Research Unit in Evaluation of Interventions at University of Bristol.

Competing interests None declared.

Patient consent for publication Not required.

Provenance and peer review Not commissioned; externally peer reviewed.

Data sharing statement The School of Social and Community Medicine (SSCM), University of Bristol, has a Data Sharing Agreement (DSA; NIC-1785-X7K1V) with the HSCIC for HES Admitted Patient Care (inpatient/day case) data for the financial years 2005/2006 to 2014/2015. The purchase of these data was funded by NIHR CLAHRC West. Copyright (c) 2018, re-used with the permission of The Health and Social Care Information Centre. All rights reserved. Our data access agreement with $\mathrm{HSCIC}$ does not allow distribution of source data, but extraction and analysis scripts are available on request. This work uses data provided by patients and collected by the NHS as part of their care and support and would not have been possible without access to these data. The NIHR recognises and values the role of patient data, securely accessed and stored, both in underpinning and leading to improvements in research and care.

\section{REFERENCES}

1 Green CA, Yeates D, Goldacre A, et al. Admission to hospital for bronchiolitis in England: trends over five decades, geographical variation and association with perinatal characteristics and subsequent asthma. Arch Dis Child 2016;101:140-6.

2 NHS Digit. Hospital admitted patient care activity, 2016-17. https://digital.nhs.uk/ data-and-information/publications/statistical/hospital-admitted-patient-care-activity/ 2016-17 (accessed 23 Aug 2018).

3 Stevens CA, Turner D, Kuehni CE, et al. The economic impact of preschool asthma and wheeze. Eur Respir J 2003;21:1000-6.

4 Régnier SA, Huels J. Association between respiratory syncytial virus hospitalizations in infants and respiratory sequelae: systematic review and meta-analysis. Pediatr Infect Dis J 2013:32:820-6.

5 Henderson J, Granell R, Heron J, et al. Associations of wheezing phenotypes in the first 6 years of life with atopy, lung function and airway responsiveness in mid-childhood. Thorax 2008;63:974-80.

6 Lipsitch M, Tchetgen Tchetgen E, Cohen T. Negative controls: a tool for detecting confounding and bias in observational studies. Epidemiology 2010;21:383-8.

7 Fauroux B, Simões EAF, Checchia PA, et al. The burden and long-term respiratory morbidity associated with respiratory syncytial virus infection in early childhood. Infect Dis Ther 2017;6:173-97.

8 Koponen $\mathrm{P}$, Helminen M, Paassilta $\mathrm{M}$, et al. Preschool asthma after bronchiolitis in infancy. Eur Respir J 2012;39:76-80.

9 Mochizuki H, Kusuda S, Okada K, et al. Palivizumab prophylaxis in preterm infants and subsequent recurrent wheezing. six-year follow-up study. Am J Respir Crit Care Med 2017; 196:29-38

10 O'Brien KL, Chandran A, Weatherholtz R, et al. Efficacy of motavizumab for the prevention of respiratory syncytial virus disease in healthy Native American infants: a phase 3 randomised double-blind placebo-controlled trial. Lancet Infect Dis 2015;15:1398-408.

11 Kuehni CE, Strippoli MP, Low N, et al. Wheeze and asthma prevalence and related health-service use in white and south Asian pre-schoolchildren in the United Kingdom. Clin Exp Allergy 2007;37:1738-46.

12 Richiardi L, Barone-Adesi F, Pearce N. Cancer subtypes in aetiological research. Eur $J$ Epidemiol 2017;32:353-61.

13 Dumas 0, Mansbach JM, Jartti T, et al. A clustering approach to identify severe bronchiolitis profiles in children. Thorax 2016:71:712-8. 